\title{
The Association between Montreal Cognitive Assessment Memory Scores and Hippocampal Volume in a Neurodegenerative Disease Sample
}

\author{
Aaron Ritter*, Nanako Hawley, Sarah J. Banks and Justin B. Miller \\ Cleveland Clinic Lou Ruvo Center for Brain Health, Las Vegas, NV, USA
}

Accepted 20 March 2017

\begin{abstract}
Despite widespread use, there have been few investigations into the neuroanatomical correlates of the Montreal Cognitive Assessment (MoCA). In a sample of 138 consecutive patients presenting with cognitive complaints, we report significant correlations between lower MoCA memory scores and smaller hippocampal volumes $(r=0.36-0.41, p<0.001)$. We also report that the newly devised memory index score, designed to better capture encoding deficits than the standard delayed recall score, was not significantly better for predicting hippocampal volume. These initial results suggest that poor performance on the MoCA's memory section should prompt further evaluation for hippocampal atrophy.
\end{abstract}

Keywords: Alzheimer's disease, dementia, hippocampus, magnetic resonance imaging, neuropsychological test

\section{INTRODUCTION}

The Montreal Cognitive Assessment (MoCA) [1] is a widely-used screening tool for cognitive impairment in Alzheimer's disease (AD) and other forms of dementia. An advantage of the MoCA over other screening tests is that performance can be deconstructed into cognitive domains, providing a more granular view of an individual's cognition $[2,3]$ that may be used to inform the initial diagnostic considerations of patients presenting with cognitive complaints. Of particular relevance in distinguishing $\mathrm{AD}$ from other dementia syndromes is the memory profile. AD is commonly associated with atrophy of the hippocampus and related mesial temporal structures that are critical for encoding new information $[4,5]$. On cognitive testing this manifests as poor

${ }^{*}$ Correspondence to: Aaron Ritter, Cleveland Clinic Lou Ruvo Center for Brain Health, 888 W. Bonneville Avenue, Las Vegas, NV 89106, USA. Tel.: +1 702484 6000; Fax: +1 702483 6039; E-mail: rittera@ccf.org. recall of previously learned information with little or no benefit from retrieval cues [6]. This memory profile, commonly described as "amnestic type" memory loss, distinguishes AD from other dementia syndromes such as Lewy body disease and vascular cognitive impairment, in which recognition memory typically exceeds free recall (i.e., memory performance improves with cues) [7, 8]. Previous work assessing the association between hippocampal volumes and individual memory processes using standard neuropsychological measures has found robust associations with estimates of both learning and recall [9-11], as well as, recognition of previously learned material $[12,13]$. Despite widespread use, there has been relatively little research into the neuroanatomical correlates associated with memory performance on the MoCA [14-16]. Only one prior study has investigated the ability of MoCA to identify different memory profiles, finding good utility in its ability to distinguish AD from Huntington's disease [17]. 
As currently constructed, the MoCA's Delayed Recall score (DR) only awards points for spontaneous recall, resulting in a metric that is limited by a restricted range (0-5) and an inability to distinguish between deficits in encoding from deficits in retrieval, even though cued recall and recognition trials can be administered. In an effort to more effectively distinguish impairments in memory encoding from retrieval, the Memory Index Score (MIS) has been developed, which incorporates performance on these additional cued recall and recognition trials [18]. In developing the MIS, Julayanont et al. [18] found that this score was better at predicting cognitive decline in mild cognitive impairment than the total score. Given the integration of cued recall and recognition, the MIS may show stronger associations with hippocampal volume than the DR score, which would thus enhance the utility of the MoCA for differentiating $\mathrm{AD}$ from other types of dementias.

The primary aim of the present paper is to explore the extent to which the two MoCA memory scores (i.e., DR and MIS) relate to hippocampal volume-a well-established correlate of memory encoding dysfunction [19]—in a sample of patients presenting with cognitive complaints. Given the wider range of possible scores and potential to isolate impairments in memory retrieval, it was hypothesized that the MIS would show stronger positive associations with hippocampal volume than simple DR scores.

\section{MATERIALS AND METHODS}

\section{Participants}

This study was conducted in accordance with guidelines on human experimentation and approved by the Cleveland Clinic institutional review board. Data were obtained from records of 138 consecutive patients who had magnetic resonance imaging (MRI) with automated volumetric analysis (NeuroQuant $\odot$ ) and cognitive screening as part of routine clinical care at an outpatient neurology clinic specializing in neurodegenerative disorders. Approximately $70 \%$ of patients seen in this neurology clinic are diagnosed with probable AD. Additional diagnoses include frontotemporal dementias, Parkinson's disease and atypical Parkinsonian syndromes, vascular cognitive impairment, Lewy body disease, mild cognitive impairment, and subjective cognitive impairment. The mean age of participants was $70.7(\mathrm{SD}=8.2$; range $=52-88$ years) and a mean of 15.2 years of education $(\mathrm{SD}=2.7$; range $=10-22)$. The analyzed sample was $50.7 \%$ male and predominantly Caucasian $(89.9 \%)$, with a mean estimated premorbid intelligence of $102.2(\mathrm{SD}=11.7)$. The average interval between the cognitive screening and MRI was 3.5 months $(\mathrm{SD}=4.3)$. As the standard of care in our clinic includes cognitive screening during the initial consult before any additional studies are ordered, the majority of patients in the present sample completed their cognitive screening prior to obtaining an MRI.

\section{Measures}

The MoCA is a brief screening measure that takes approximately 10 minutes to complete. Standard administration consists of 12 individual tasks grouped into seven cognitive domains: 1) visuospatial/executive; 2) naming; 3) attention; 4) language; 5) abstraction; 6) memory; and 7) orientation. Task performance is summed generating both domain scores and a total score. An educational correction of one point is added to the total score for individuals with 12 years of education or less. Validated use of the MoCA is based on interpretation of the total score, with a score of 26 or less indicative of cognitive impairment [1].

The memory section of the MoCA consists of two initial learning trials of a five-word list. The delay condition is completed several minutes later, beginning first with free recall, followed by provision of categorical cues for unrecalled items, and then multiple choice cues for items not recovered with a categorical cue. The DR score is calculated by adding one point for each word spontaneously recalled and ranges from 0 to 5 . The MIS incorporates the cued recall and recognition trials and awards three points for each word spontaneously recalled, two points for each word recovered with category cue, and one point for each word correctly identified during multiplechoice recognition. The range of the MIS is 0 to 15 .

MRI sequences were obtained on the same Siemens TIM Verlo 3T scanner. Volumetric data were generated by NeuroQuant ${ }^{\odot}$, an automated program approved by the Food and Drug Administration for clinical measurement of brain volume in human subjects [20]. NeuroQuant ${ }^{\circledR}$ also estimates the percentage of total hemispheric volume for each brain region. This study specifically looked at the hippocampus because of its importance as a biomarker in AD. The thalamus, which is not associated with accelerated atrophy in $\mathrm{AD}$, was used as a secondary area to ensure that any structural-performance relationships were not simply due to generalized atrophy. 


\section{Statistical analysis}

Partial correlation coefficients, accounting for age, education, and premorbid intelligence, were calculated to determine the relationship between hippocampal volume (calculated as percentage of total hemispheric volume) and DR and MIS scores. Fisher's R-to-Z transformation for dependent correlations was used to compare these correlations. Several separate linear regression models were also fit using age, education, and estimated premorbid intelligence as predictors and hippocampal volume as the outcome. The MoCA DR and MIS were then added individually to each base model to determine the added predictive value of including memory scores for predicting hippocampal volume. Given findings from previous research suggesting material specificity [9], separate models were fit for both the left and the right hemisphere. Model performance was evaluated by the change in R-squared values from adding the memory indices to the base model, and the influence of the MIS or DR on hippocampal volume was assessed via the standardized regression coefficient. To ensure findings were not a function of generalized brain atrophy, regression analyses were repeated using thalamic volumes.

\section{RESULTS}

Demographic data for the sample are presented in Table 1 , and means and standard deviations and partial correlations for the MoCA scores and hippocampal volumes are presented in Table 2. Partial correlations between the DR score and hippocampal volume were positive (weak to moderate) such that lower scores were associated with smaller hippocampal volumes (Left: $r=0.39, p<0.001$; Right: $r=0.36, p<0.001)$. Partial correlations between the MIS and hippocampal volumes were also positive (weak to moderate) (Left: $r=0.40, p<0.001$; Right: $r=0.39, p<0.001)$. Fisher's R-to-Z transformations
Table 1

Demographic details of cohort

\begin{tabular}{lccccc}
\hline & $\mathrm{N}$ & Mean & $\%$ & SD & Range \\
\hline Age (years) & 138 & 70.69 & & 8.16 & $52-88$ \\
Education & 138 & 15.18 & & 2.73 & $10-22$ \\
Female & 68 & & 49.3 & & \\
Caucasian & 124 & & 89.9 & & \\
African American & 8 & & 5.8 & & \\
Hispanic & 3 & & 2.2 & & \\
\hline
\end{tabular}

indicate that although qualitatively larger, the correlations between hippocampal volume and the MIS were not statistically different than those for DR and hippocampal volume (Left: $\mathrm{z}=0.27, p=0.39$; Right: $\mathrm{z}=0.81, p=0.21)$. Neither the MIS nor DR was significantly correlated with left or right thalamic volume.

Left and right hippocampal volumes as a function of DR score are presented in Fig. 1 and the results of the regression are presented in Table 3. For each model fit, adding either the DR or the MIS score significantly increased the proportion of variance accounted for beyond demographic factors alone. The MIS was similarly predictive of both left and right hippocampal volume, and was marginally better at predicting hippocampal volume than DR. Notably, the only significant predictors were age and the memory score (either the DR or the MIS); premorbid intelligence and education were not significant in any model. Adding memory scores to the base models did not improve model fit for predicting thalamic volume.

\section{DISCUSSION}

Findings from the present study support the hypothesis that memory scores on the MoCA are correlated with hippocampal volume in a mixed clinical sample of patients presenting with cognitive complaints. Although correlations were moderate to weak and only accounted for $12 \%$ of variance when

Table 2

Descriptive statistics and partial correlations accounting for age, education, and premorbid intelligence

\begin{tabular}{lccccc}
\hline & MoCA & MoCA & MoCA & Left & Right \\
& Total & DR & MIS & Hippocampus & Hippocampus \\
\hline MoCA Total & $21.9(5.0)$ & 0.71 & 0.70 & 0.39 & 0.41 \\
MoCA DR & - & $1.9(1.7)$ & 0.89 & 0.39 & 0.36 \\
MoCA MIS & - & - & $8.9(4.1)$ & 0.40 & 0.39 \\
Left Hippocampus & - & - & - & $0.23(0.03)$ & 0.71 \\
Right Hippocampus & - & - & - & - & $0.25(0.04)$ \\
\hline
\end{tabular}

Means and standard deviations are presented along the diagonal. Hippocampal volumes reported as a percentage of the total intracranial volume. All correlations significant at the $p<0.001$ level. 


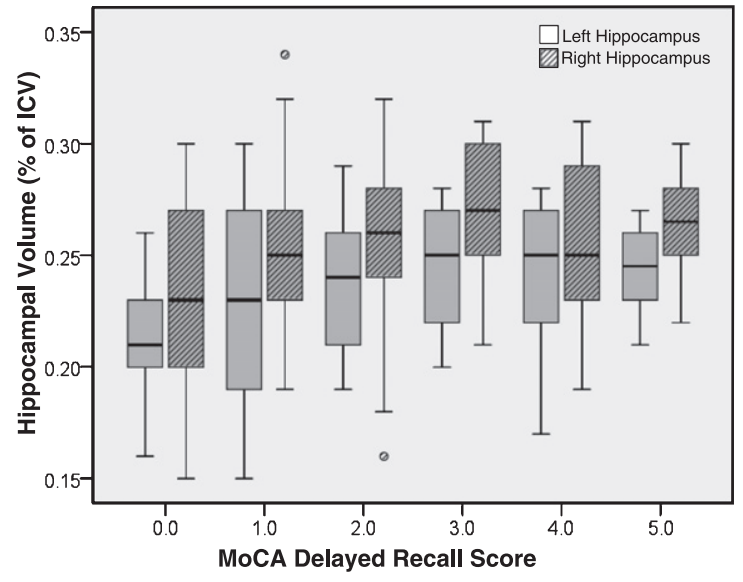

Fig. 1. Hippocampal volume (as a percentage of intracranial volume) by MoCA delayed recall score.

\section{Table 3}

Regression results predicting structural volume using demographic variables and MoCA memory scores

\begin{tabular}{lcccc}
\hline Side & Predictor & $\mathrm{R}^{2}$ Change & Adjusted $\mathrm{R}^{2}$ & Beta \\
\hline Left Hippocampal & $\mathrm{DR}$ & 0.12 & 0.28 & 0.37 \\
Volume & MIS & 0.13 & 0.29 & 0.38 \\
Right Hippocampal & $\mathrm{DR}$ & 0.11 & 0.26 & 0.35 \\
Volume & MIS & 0.13 & 0.28 & 0.38 \\
\hline
\end{tabular}

$\mathrm{R}$-squared change values reflect addition of the memory predictor to the base demographic model. All R-squared change values and standardized regression coefficients significant at the $p<0.001$ level.

added to regression models, the same associations were not seen with thalamic volume. Therefore, it is unlikely that this association is merely a product of global brain atrophy. Thus, when low memory scores are encountered in clinic this may be indicative of hippocampal atrophy and should prompt further evaluation to explore potential causes, as neither low memory scores or hippocampal atrophy in isolation of other clinical indicators is pathognomonic of a particular etiology.

An unexpected finding from this study is that that there was little evidence to suggest an advantage of the MIS as compared to the basic DR score. Although correlations between the MIS and hippocampal volumes were stronger than the correlations between DR and hippocampal volume, and the MIS accounted for a marginally greater proportion of variance in hippocampal volumes, the magnitude of the observed differences was not significant. This is likely because calculation of the MIS heavily weights free recall performance. It is therefore not surprising that there is little difference between the two indices. Future directions should explore the possibility of calculating a dedicated recognition score, which may be most useful when free recall is at floor levels. This would not only align the MoCA more closely with standard neuropsychological memory tests, but may also prove more sensitive to mesial temporal dysfunction than current primary memory indices from the MoCA.

This study has several limitations, most notably the retrospective nature of the data collection. The diagnostic diversity of the cohort can also be seen as a weakness; however, the clinical heterogeneity of the current sample is likely to reflect that which is typically encountered in clinical practice, the inclusion of a variety of diseases in the cohort precludes refined study of these relationships in specific diseases. Despite the availability of diagnostic information, both the MoCA score and hippocampal volume were used in the clinical-decision making process, preventing a study of specific patient groups using the present dataset. Disease specific investigations are an additional area for future study. Another limitation is the length of time that passed between imaging and cognitive screening, which for some individuals (e.g., those with rapidly progressing dementia) may have introduced unaccounted for error variance in our analyses. To the extent possible, future studies should make every effort to minimize the interval between studies (preferably even same-day).

\section{Conclusion}

The utility of the MoCA as a screening tool has been well established, and the present paper extends the evidence-base, demonstrating the association of MoCA with neuroanatomical structure in patients presenting with cognitive complaints. Although in this study there was minimal advantage to using the MIS over the DR score and calculating both values would be redundant in many circumstances, there may still be some utility in calculating the MIS when a patient is unable to recall any words during the free recall trial. There remains an ongoing need to expand upon the present study and explore ways in which an individual's pattern of performance on the eight cognitive domains included in the MoCA can be used to inform the differential diagnosis of patients presenting with cognitive impairment.

\section{ACKNOWLEDGMENTS}

This work was supported in part by a Center of Biomedical and Research Excellence (COBRE) 
grant (reference number: 1P20GM109025-01A1). The funding source had no role in the study design, collection, or interpretation of the data.

Authors' disclosures available online (http://j-alz. com/manuscript-disclosures/16-1241r2).

\section{REFERENCES}

[1] Nasreddine ZS, Phillips NA, Bedirian V, Charbonneau $\mathrm{S}$, Whitehead V, Collin I, Cummings JL, Chertkow H (2005) The Montreal Cognitive Assessment, MoCA: A brief screening tool for mild cognitive impairment. $J$ Am Geriatr Soc 53, 695-699.

[2] Lam B, Middleton LE, Masellis M, Stuss DT, Harry RD, Kiss A, Black SE (2013) Criterion and convergent validity of the Montreal cognitive assessment with screening and standardized neuropsychological testing. J Am Geriatr Soc 61, 2181-2185.

[3] Vogel SJ, Banks SJ, Cummings JL, Miller JB (2015) Concordance of the Montreal cognitive assessment with standard neuropsychological measures. Alzheimers Dement (Amst) 1, 289-294.

[4] Weintraub S, Wicklund AH, Salmon DP (2012) The neuropsychological profile of Alzheimer disease. Cold Spring Harb Perspect Med 2, a006171.

[5] Sarazin M, Berr C, De Rotrou J, Fabrigoule C, Pasquier F, Legrain S, Michel B, Puel M, Volteau M, Touchon J, Verny M, Dubois B (2007) Amnestic syndrome of the medial temporal type identifies prodromal AD: A longitudinal study. Neurology 69, 1859-1867.

[6] Tounsi H, Deweer B, Ergis AM, Van der Linden M, Pillon B, Michon A, Dubois B (1999) Sensitivity to semantic cuing: An index of episodic memory dysfunction in early Alzheimer disease. Alzheimer Dis Assoc Disord 13, 38-46.

[7] Mormont E, Laurier-Grymonprez L, Baisset-Mouly C, Pasquier F (2003) The profile of memory disturbance in early Lewy body dementia differs from that in Alzheimer's disease. Rev Neurol (Paris) 159, 762-766.

[8] Grober E, Hall C, Sanders AE, Lipton RB (2008) Free and cued selective reminding distinguishes Alzheimer's disease from vascular dementia. J Am Geriatr Soc 56, 944-946.

[9] Bonner-Jackson A, Mahmoud S, Miller J, Banks SJ (2015) Verbal and non-verbal memory and hippocampal volumes in a memory clinic population. Alzheimers Res Ther 7, 61 .

[10] Deweer B, Lehericy S, Pillon B, Baulac M, Chiras J, Marsault C, Agid Y, Dubois B (1995) Memory disorders in probable Alzheimer's disease: The role of hippocampal atrophy as shown with MRI. J Neurol Neurosurg Psychiatry 58, 590-597.

[11] Hirni DI, Kivisaari SL, Monsch AU, Taylor KI (2013) Distinct neuroanatomical bases of episodic and semantic memory performance in Alzheimer's disease. Neuropsychologia 51, 930-937.

[12] Manns JR, Hopkins RO, Reed JM, Kitchener EG, Squire LR (2003) Recognition memory and the human hippocampus. Neuron 37, 171-180.

[13] Wolk DA, Dickerson BC (2011) Fractionating verbal episodic memory in Alzheimer's disease. Neuroimage 54, 1530-1539.

[14] Paul R, Lane EM, Tate DF, Heaps J, Romo DM, Akbudak E, Niehoff J, Conturo TE (2011) Neuroimaging signatures and cognitive correlates of the montreal cognitive assessment screen in a nonclinical elderly sample. Arch Clin Neuropsychol 26, 454-460.

[15] Zhang YW, Zhang JQ, Liu C, Wei P, Zhang X, Yuan QY, Yin XT, Wei LQ, Cui JG, Wang J (2015) Memory dysfunction in type 2 diabetes mellitus correlates with reduced hippocampal CA1 and subiculum volumes. Chin Med J (Engl) 128, 465-471.

[16] Gupta M, King KS, Srinivasa R, Weiner MF, Hulsey K, Ayers CR, Whittemore A, McColl RW, Rossetti HC, Peshock RM (2015) Association of 3.0-T brain magnetic resonance imaging biomarkers with cognitive function in the Dallas Heart Study. JAMA Neurol 72, 170-175.

[17] Van Liew C, Santoro MS, Goldstein J, Gluhm S, Gilbert $\mathrm{PE}$, Corey-Bloom J (2016) Evaluating recall and recognition memory using the Montreal Cognitive Assessment: Applicability for Alzheimer's and Huntington's diseases. Am J Alzheimers Dis Other Demen 31, 658-663.

[18] Julayanont P, Brousseau M, Chertkow H, Phillips N, Nasreddine ZS (2014) Montreal Cognitive Assessment Memory Index Score (MoCA-MIS) as a predictor of conversion from mild cognitive impairment to Alzheimer's disease. J Am Geriatr Soc 62, 679-684.

[19] Petersen RC, Jack CR Jr, Xu YC, Waring SC, O'Brien PC, Smith GE, Ivnik RJ, Tangalos EG, Boeve BF, Kokmen E (2000) Memory and MRI-based hippocampal volumes in aging and AD. Neurology 54, 581-587.

[20] Ochs AL, Ross DE, Zannoni MD, Abildskov TJ, Bigler ED (2015) Comparison of automated brain volume measures obtained with NeuroQuant and FreeSurfer. J Neuroimaging 25, 721-727. 